\title{
Research on the impact of payoff reflection mechanism on cooperation evolution
}

\author{
Bin Wang \\ Central South University \\ Bo Huang \\ Central South University \\ Jinfang Sheng ( $\sim$ jfsheng@csu.edu.cn ) \\ Central South University \\ Qiangqiang Dong \\ Central South University \\ Wenjun Kang \\ Central South University
}

\section{Research Article}

Keywords: cooperation evolution, payoff reflection mechanism

Posted Date: December 15th, 2020

DOI: https://doi.org/10.21203/rs.3.rs-125743/v1

License: (c) (1) This work is licensed under a Creative Commons Attribution 4.0 International License. Read Full License 


\title{
Research on the impact of payoff reflection mechanism on cooperation evolution
}

\author{
Bin Wang ${ }^{1}$, Bo Huang ${ }^{1}$, Jinfang Sheng ${ }^{1, *}$, Qiangqiang Dong ${ }^{1}$, and Wenjun Kang ${ }^{1}$ \\ ${ }^{1}$ School of Computer Science and Engineering, Central South University, Changsha, 410006, China \\ *jfsheng@csu.edu.cn
}

\begin{abstract}
Taking into account the results of historical behavior in real life on the choice of strategy, we proposes a payoff reflection mechanism. In the process of cooperation evolution, the game individuals will judge whether to learn each other's strategies based on the historical payoff of himself and his neighbors, that is, the strategy learning is affected by the historical payoff. If the current strategy can bring greater payoff in historical behavior, the game players will change the current strategy with less possibility. For this reason, the historical payoff reference coefficients $w$ and $u$ of the game individuals and their neighbors are proposed to measure the degree of reference of game individuals to their own and neighbors' historical payoff. The memory interval length is expressed by $\mathrm{M}$. The experimental results show that the payoff reflection mechanism can greatly improve the cooperation level of the group. However, the reference rate of historical payoff is not the bigger the better. The strength of memory ability and the level of betrayal temptation will affect the optimal value of $w$ and $u$.
\end{abstract}

\section{Introduction}

Game theory is the subject of optimizing game strategy by studying individual behaviors of individuals in the competition. Game theory has many applications in economics, sociology, biology, politics, computer science, military strategy and other sciences $^{1,2}$. Cooperative behavior is a common phenomenon in biological ${ }^{3}$ and social systems ${ }^{4}$. Understanding the emergence and maintenance of cooperative behavior attracts a lot of scholars' attention ${ }^{5,6}$. Evolutionary game theory provides a powerful theoretical framework for the study of the emergence and maintenance of cooperative behavior in a group of selfish individuals ${ }^{7-9}$. Common game models include prisoner's dilemma game (PDG) $)^{10}$, snowdrift dilemma game $(\mathrm{SDG})^{11}$ and stag-hunt game $(\mathrm{SHG})^{12}$.

In 1992, Nowak and May pioneered the study of the evolution of cooperation on the grid network ${ }^{13}$. Nowak and May introduced cyberspace to evolutionary game for the first time, and opened up new research ideas and methods of evolutionary game. With the development of evolutionary game, the influence of different network structures on cooperative behavior has been investigated, such as small-world networks ${ }^{14,15}$, scale-free networks ${ }^{16,17}$ and interdependent networks ${ }^{18}$. In addition, many mechanisms have been proposed to promote cooperation, such as neighborhood diversity ${ }^{19}$, environmental factor ${ }^{20}$, link weight $^{21,22}$, reputation ${ }^{23}$, punishment ${ }^{24}$ and reward ${ }^{25}$, etc.

Memory effect also attracts many scholars' attention ${ }^{26-28}$. In the spatial cooperation evolution model proposed by Nowak, the next step strategy of game individuals is determined by the payoff of the current time step. That is to say, game payoff affects the choice of game strategy completely. The influence of historical payoff on the evolution of cooperation has been studied by many scholars, and relevant models have been proposed constantly. Wang et al. proposed an improved evaluation mechanism of spatial prisoner's dilemma memory fitness based on rule lattice ${ }^{29}$. The current round of game proceeds and the previous round of earnings combination were used as individual fitness to study the effect of this fitness on the level of cooperation. Based on the payoff, they studied the role of memory in promoting the cooperation level of selfish players. Based on the work of Wang. Geng et al. studied the prisoner's dilemma game model with volunteers ${ }^{30}$, and they proposed the adjustment parameters of historical payoff. Deng et al. also studied the traditional prisoner's dilemma game model based on similar payoff model ${ }^{31}$.

In related work, we can found that historical payoff will promote the level of cooperation among evolutionary groups, and explain the impact of historical payoff in the evolution of cooperation. But in real life, we can learn from the experience of recent time steps. When a game player memorize the history, the content of his memory is also varied. Not only will he remember the payoff, but also the strategies to obtain this payoff. Therefore, in this paper, the historical game strategy and the corresponding historical game payoff are recorded in the memory interval of the game players. In real life, experience plays an important role in making decisions. When different strategies are needed to choose, the different results of different choices in the memory will have an impact on the current choice. People tend to reflect on the historical behavior and choose the strategy to maximize their own payoff. Therefore, this paper proposes a payoff reflection mechanism. The game individual will reflect when choosing the current strategy, and calculate the difference between the current strategy's payoff in memory and the payoff 
of another strategy as a reference. Obviously, if the current strategy's payoff in memory is higher, the probability of the game player changing his strategy in the current time step should be lower.

\section{Results}

\section{The impact of payoff reflection mechanism on the level of cooperation}

In real life, the reflection behavior on historical decisions and their results can be divided into three categories: (1) Self-reflection of selfish individuals, that is, individuals will only reflect on their own historical behavior; (2) Self-reflection of conformity individuals, that is, individuals will only reflect on the historical behavior of others; (3) Self-reflection of ordinary individuals, that is, individuals will not only reflect on their historical behavior. It will also be influenced by other individuals' historical behavior. For the above three reflective behaviors, we can adjust the reference coefficient of reflective payoff to correspond to one of them. Under the premise of $0 \leq w, u<1$, when $w \neq 0$ and $u=0$, game players only reflect on their own historical payoff; when $w=0$ and $u \neq 0$, the game players only reflect on the historical payoff of the strategy learning target individuals; when $w \neq 0$ and $u \neq 0$, the game players reflect on the historical payoff of themselves and their neighbors. How the group cooperation level $\mathrm{Fc}$ varies with the increase of betrayal temptation $\mathrm{b}$ under different memory interval length $\mathrm{M}$ in three reflective behaviors is shown in fig. 1. The payoff reflection parameters in fig. 1(a), fig. 1(b) and fig. 1(c) are $w=0.1, u=0$ (selfish individuals), $w=0, u=0.1$ (conformity individuals) and $w=0.6, u=0.4$ (ordinary individual). When $M=0$, the game individuals has no memory ability, they choose their neighbor randomly and only calculates the probability of learning the opponent's strategy based on the game proceeds of the current time step. At this time, the model returns to the traditional form, and its corresponding cooperation level change curve is used as the comparative data after introduction the payoff reflection mechanism. This section mainly studies on square lattice with the size $50 \times 50$. We rendered experiments on Feiteng Server provided by Kirin Operating System, Feiteng 1500A CPU, 32G memory and 2TB hard disk.

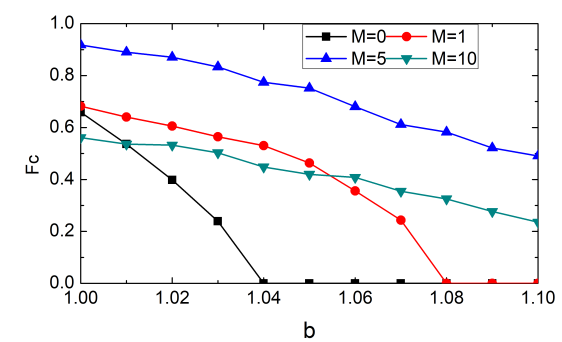

(a) selfish individuals

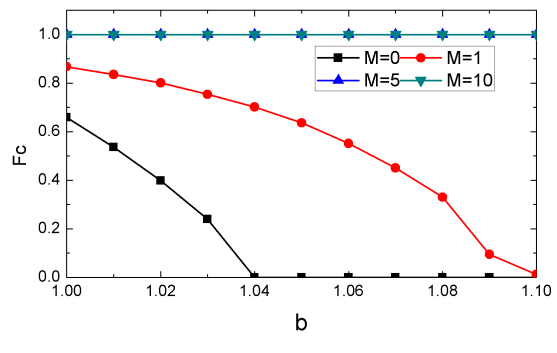

(b) conformity individuals

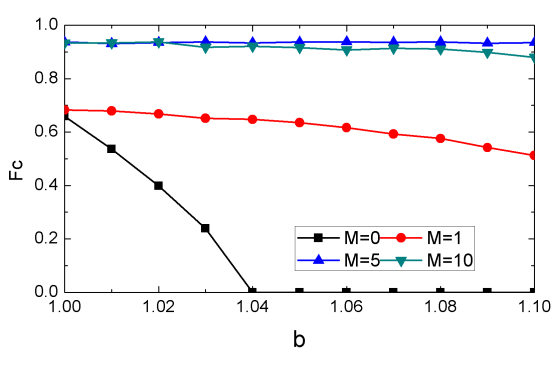

(c) ordinary individuals

Figure 1. Influence of payoff reflection mechanism on cooperation level.

In fig. 1(a), when $M=0$, with the increase of betrayal temptation $\mathrm{b}$, the level of group cooperation $F_{C}$ decreases rapidly. When betrayal temptation $b=1.04$, there are no cooperators in the evolutionary group. When $M \neq 0$, although the level of group cooperation $F_{C}$ will still decrease with the increase of betrayal temptation b, the rate of decline is significantly reduced, and there will still be a certain proportion of collaborators in the evolutionary group under the larger betrayal temptation. When $b \geq 1.01$, the cooperation level curve based on the payoff reflection mechanism is always on the top of the change curve corresponding to $M=0$, especially when $M=5$, the overall level of cooperation has been significantly improved. But when $M=10, b<1.01$, the level of group cooperation is lower than that of the traditional model. In the reflective payoff model of selfish individuals, the payoff reflection mechanism can have a positive impact on the level of cooperation, but when the temptation of betrayal is small and the memory ability is strong, the payoff reflection mechanism will inhibit the level of group cooperation.

The variation curve of the cooperation level of the conformity individual is shown in fig. 1(b). It can be seen from the figure that the payoff reflection mechanism has an obvious effect on the improvement of cooperation level, and at the same time, it can also be seen that the cooperation level is different from that of selfish individuals. Comparing fig. 1(a) and fig. 1(b), we can found that even if the memory ability of a game individual is weak, that is, when $M=1$, the payoff reflection mechanism of the conformity individuals will significantly improve the level of group cooperation. When the memory ability is enhanced, there is no case of cooperation below the level of no memory, and the strategy selection of the group can all be cooperation. In the payoff reflection model of the conformity individual, the payoff reflection mechanism has a significant effect on the level of cooperation. When a game player has memory ability, the cooperation level of the group will be improved regardless of the memory ability. When the memory ability is strong, the group cooperation level can reach $100 \%$.

The influence curve of ordinary individual's payoff reflection behavior on group cooperation level is shown in fig. 1(c). 
Comparing the previous two reflective behaviors, we can see that the variation curve of cooperation level under different memory interval length of general individuals is basically between the previous two models. When the memory ability is relatively strong, the general group cooperation level is higher than that of selfish individuals. Moreover, the increase of betrayal and attraction has little effect on the cooperation level of the group. When the length of memory interval is long, the cooperation level tends to be almost a straight line, but the cooperation level will not reach $100 \%$. When the memory ability is weak, although the level of group cooperation will decrease, the downward trend will be slower. In the payoff reflection model of general individuals, the payoff reflection mechanism also significantly increases the cooperation level of the evolved group and slows the decline of the group cooperation level. When the memory ability is strong, the cooperation level will hardly decrease with the increase of the temptation of betrayal.

Through experimental data analysis, we can see that no matter which kind of reflection mode, the payoff reflection mechanism has an impact on the change of the group cooperation level. When the payoff reflection parameters and the length of the memory interval are appropriate, the cooperation level of the group will be significantly improved. Especially when the memory ability is strong, the reflexive behavior of the conformity individuals can make the cooperation level of the group reach $100 \%$. However, game individuals will not rely solely on their own or other's memories to reflect. Therefore, based on the consideration of the irrational behavior mode of the game individual, in the next simulation experiments, only the payoff reflection mechanism of general individuals is studied. In order to further studies the situation that the level of group cooperation changes with the increase of betrayal temptation after introducing the payoff reflection mechanism of general individuals, as shown in fig. 2 . When the game individual has no memory ability and the betrayal temptation $\mathrm{b}$ is small, the level of group cooperation rapidly reduces to 0 . When the game individual have the memory ability, even if the temptation of betrayal increases substantially, the cooperation level of the group will remain at a higher level. When the betrayal temptation $b$ is greater than a certain value, the cooperation level of the group will be reduced to 0 , but it can be seen that the stronger the memory ability, the greater the temptation of betrayal when the level of group cooperation is reduced to 0 . When the temptation of the betrayal is greater, the effect of the payoff reflection mechanism on the group cooperation level will be better.

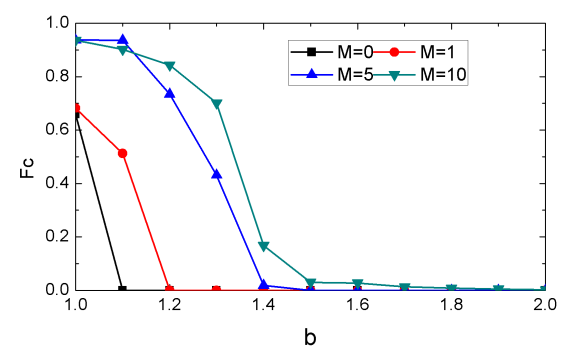

Figure 2. The influence of general individual's payoff reflection mechanism on the level of cooperation under high betrayal temptation.

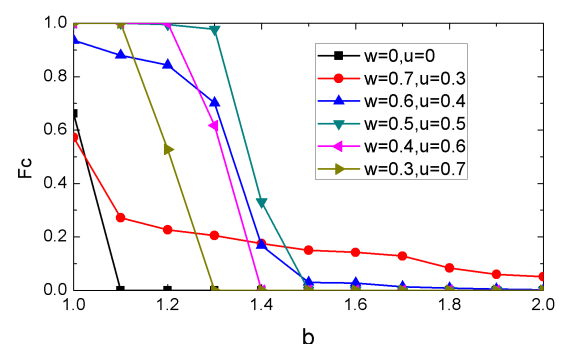

Figure 3. The impact of payoff reflection parameters on the level of cooperation

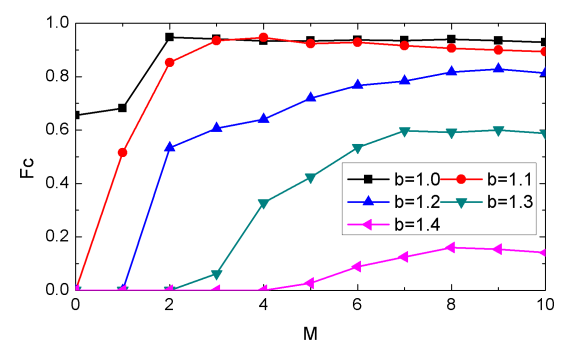

Figure 4. Influence of memory interval length on cooperation level.

\section{The influence of $w$ and $u$ on cooperation level}

In the previous subsection, it has been found that the payoff reflection mechanism has an obvious effect on the improvement of the cooperation level of evolutionary games. Taking into account the reflection behavior of irrational individuals, we decide to use the ordinary individual payoff reflection model to carry out the experiment. Next, we mainly study the impact of parameter changes in the payoff reflection model of ordinary individuals on the level of cooperation, which is mainly divided into two parts: The influence of self-reflection parameter $\mathrm{w}$ and learning reflection parameter $\mathrm{u}$ on the level of group cooperation, and the influence of memory interval length $\mathrm{M}$ on the level of group cooperation.

In daily life, different individuals have different reflective behaviors, or refer to other individuals' historical behavior more, or refer to their own historical payoff more, or self-reflection and learning reflection are mixed. In addition to the different weights of self-reflection and learning reflection, different individual's historical behavior has different influence on current behavior. Based on the above considerations, the relationship between the payoff reflection parameter $\mathrm{w}$ and $\mathrm{u}$ is set as $w+u=1$. On this premise, the influence of changes in the self-reflection parameter $\mathrm{w}$ and learning reflection parameter $\mathrm{u}$ on the level of group cooperation is studied. The change chart of cooperation level is shown in fig. 3 , where $M=10$, when $w=0$ and $u=0$, it means that the influence of historical payoff is not taken into account.

We can see that with the self-reflection parameters $w$ increased from 0 to 0.5 , the change curve of cooperation level moves to the right of the whole. that is, the larger the self-reflection parameter $\mathrm{w}$ is, the higher the cooperation level is. When w continues 
to increase from 0.5 , the overall cooperation level curve shows a trend of moving to the left and down. When the betrayal temptation is relatively low, the larger the self-reflection parameter $\mathrm{w}$ is, the lower the cooperation level will be. However, the cooperation level of the group will not drop to 0 , and the higher $\mathrm{w}$ is, the higher the cooperation level will be. The change of payoff reflection parameters $\mathrm{w}$ and $\mathrm{u}$ will have a great impact on the level of cooperation. Under the premise that the sum of the self-reflection parameter $\mathrm{w}$ and the learning reflection parameter $\mathrm{u}$ is certain, when the temptation to betray $\mathrm{b}$ is small, the increase in the reference weight of self-reflection will cause the group cooperation level to rise first and then decrease. When the temptation of betrayal $b$ is large, a higher proportion of self-reflection will not reduce the level of group cooperation to 0 . For different temptations of betrayal, there are different optimal parameter proportions. As can be seen in fig 3 , when the temptation of betrayal is relatively small, the self-reflection parameter $\mathrm{w}$ and the learning reflection parameter $\mathrm{u}$ are both 0.5 , which makes the group cooperation level present an optimal state. However, in real life, the general individual cannot achieve the same proportion of the two. Considering that the individual is selfish and irrational, the self-reflection reference coefficient $\mathrm{w}$ is set to 0.6 , and the coefficient $\mathrm{u}$ for learning reflection is 0.4 .

\section{The impact of $M$ on cooperation level}

This subsection studies the influence of the change of memory interval length $\mathrm{M}$ on the group cooperation level. The change of cooperation level is shown in fig 4 , where $w=0.6, u=0.4$. When $M=0$, it means that the game player has no memory ability. Only the current time step's payoff is considered when calculating the payoff. It can be seen that with the temptation of the betrayal increases, the overall level of cooperation is declining. When the temptation of betrayal is low, the level of group cooperation under each memory interval length can be maintained at a very high level. Even if the temptation of betrayal is large, there will still be a certain proportion of collaborators in the group with strong memory. We can see that the payoff reflection mechanism itself has a very obvious promotion effect on the cooperation level of the group when the temptation of betrayal is low, but the mechanism itself cannot prevent the trend of cooperators to decrease under high betrayal temptation.

The change curve of group cooperation level under the different temptation of betrayals all present a trend of rising first and then falling, but the trend of decline is weak. When the temptation of betrayal is low, with the enhancement of memory ability of game individual, the level of group cooperation will increase at a faster rate and then decrease slightly. However, when the temptation of betrayal is high, even if the memory ability of the game players increases gradually, the cooperation level will be as low as 0 . The differences of cooperation level under different memory interval lengths are quite obvious. According to the analysis, it can be concluded that under the condition of low betrayal temptation, the enhancement of memory ability showed a trend of first increasing and then decreasing in the improvement of group cooperation level. When the temptation of betrayal is high, only strong memory ability can effectively improve the cooperation level of the group. However, the level of cooperation will still increase first and then decrease with the enhancement of memory ability. For different temptations of betrayal, the optimal memory ability that can reach the highest level of group cooperation is also different.

\section{The influence of $\mathbf{w}, \mathrm{u}$ and $\mathrm{M}$ on the evolution of cooperation}

This subsection further studies the influence of payoff reflection parameters $\mathrm{w}, \mathrm{u}$ and the memory interval length $\mathrm{M}$ on the evolution of cooperation. Fig.5 shows the change of cooperation level Fc with the increase of Monte Carlo time steps t under different memory interval length $\mathrm{M}$ and payoff reflection parameters $\mathrm{w}$ and $\mathrm{u}$. In fig $5(\mathrm{a}), b=1.2, w=0.6, u=0.4$. In fig 5(b), $b=1.2, M=5$. In the following analysis, $F c_{\text {min }}$ is used to represent the lowest level of group cooperation level, which indicates the level of cooperation when the group cooperator cluster is formed. $F c_{\max }$ is used to represent the average level of cooperation after the evolutionary game reaches a stable state, which indicates the final cooperation level of the group.

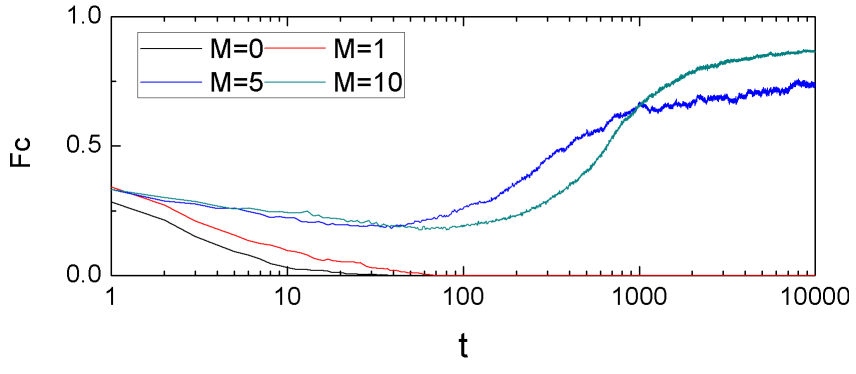

(a)

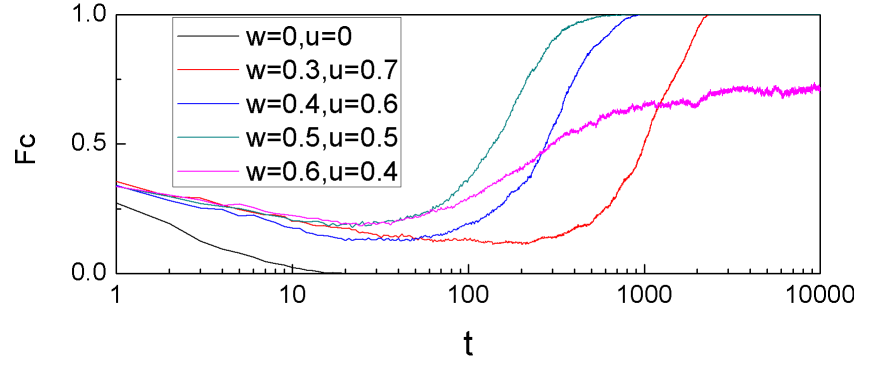

(b)

Figure 5. The influence of the parameters of the payoff reflection mechanism on the evolution of cooperation.

As can be seen from fig. 5(a), when $M=0$, the level of group cooperation rapidly decreases to 0 . When $M=1$, the level of cooperation will still decrease to 0 at a faster rate. However, the time for cooperation level to decrease to 0 increases 
significantly compared with when $M=0$, which shows that the mechanism of payoff reflection has an impact on the evolution of cooperation. With the memory ability of the game individual continues to enhance, the cooperation level of the group presents a trend of first decreasing, then increasing, and finally stabilizing. The improvement of memory ability makes the promotion effect of cooperation level brought by the payoff reflection mechanism begin to appear. Observing the change chart of cooperation level, we can also see that when the game individual memory ability of is strong, the cooperation level $F c_{m a x}$ will be higher under the condition of stable evolution. Through the influence of the change of the length of memory interval on the cooperation evolution, we can found that the cooperation level of group will increase with the enhancement of memory ability. Comparing the two evolution curves of $M=5$ and $M=10$, we can see that the difference in the value of $F c_{m i n}$ between the two is small, but the time to reach $F c_{\min }$ is significantly different. It shows that the enhancement of memory ability will hardly affect the scale of the formation of the collaborator clusters, but it will affect the formation time of the cooperative clusters. The stronger the memory ability, the longer it takes for the clusters of collaborators to form. The time for the two to reach the steady state of evolution is also different, and the game group with weaker memory ability can reach the steady state of evolution faster. In addition, we can see that the evolution curve of group cooperation formed by individuals with strong memory ability is more smooth and evolvement, and the volatility is very small. It shows that the level of group cooperation fluctuates within a small range in each time period after the formation of the collaborator cluster, and the evolution process of the group with stronger memory ability is more stable.

The evolution curve of cooperation level with the time step growth under different value of payoff reflection parameters $w$ and $u$ is shown in fig 5(b). When $w=0$ and $u=0$, it represents the traditional evolutionary model. The traditional evolutionary model does not consider the impact of historical payoff on current strategy learning. The relationship between $w$ and $u$ reflects the reference weight of game individual to historical payoff of himself and his neighbors. We can see that if the influence of historical payoff is not taken into account, the number of collaborators in the evolution group will quickly drop to 0 . With the increase of the reference weight of self-reflection, the cooperation evolution curve will gradually shift to the left. When the reference weight of self-reflection payoff is greater than that of the reference weight of historical payoff to game neighbors, the level of cooperation is significantly reduced. The larger the reference weight $u$ of neighbor's payoff reflection, the smaller the $F c_{\text {min }}$, and the larger the $t$ reaches $F c_{\min }$ and $F c_{\max }$. The historical benefit of gaming neighbors is relatively high, which will make it take longer for the evolutionary group to form a cluster of smaller cooperative to resist the temptation of betrayal from the outside, and it will also take longer for the evolutionary group to reach an evolutionary stable state. By comparing the historical payoff reference models with different weight, it can be found that when the individual have the same historical payoff reference weight to themselves and their neighbors, there is the best cooperative evolution process. At this time, the evolution group needs the least time to form a cooperative cluster that can resist the temptation of betrayal. The cluster size is relatively large, and the time for cooperative evolution to reach the stable state is also the least, that is, the evolutionary population at this time will reach the optimal stable state in the least time.

From the study of the influence of memory interval length $\mathrm{M}$ and payoff reflection parameters $w$ and $u$ on the evolution of cooperation, we can find that the payoff reflection mechanism is strongly dependent on the memory ability of game individuals. Weak memory ability can not change the trend of group evolution. When the memory ability is strong, the level of group cooperation will be significantly improved, and the evolution process will be smoother. The difference of reference weight between individuals and their neighbors will make the evolution process different, and the reference weight of their historical payoff is inversely proportional to the level of cooperation.

\section{The impact of payoff reflection on the level of cooperation in other networks}

In order to study the impact of the payoff reflection mechanism on the cooperation level based on the small world network, random network and scale-free network ${ }^{32}$, and still uses the prisoner's dilemma game mode. The average node degree of the random network is 5 . The average node degree of the small-world network is 4 , and the reconnection probability is 0.3 . The maximum node degree of the scale-free network is 20. The scale of the 3 networks is 1000 nodes. The impact of the payoff reflection mechanism on the level of group cooperation is shown in fig. 6, where fig. 6(a) is based on small world network, fig. 6(b) is based on random network, and fig. 6(c) is based on scale-free network. The length of memory interval in experiments is 5. For different network models, this section adopts the payoff reflection model of general individuals, considering both our historical payoff and the historical payoff of our neighbors. This paper mainly studies the influence of the mechanism of payoff reflection on the level of group cooperation when the weight of self-payoff reflection and learning payoff reflection is different under different network models. In this experiment, when the parameters of self-payoff reflection w and learning payoff reflection $\mathrm{u}$ are both 0 , it means that the experimental model is a tradition model and without considering the influence of historical payoff. Different values of $w$ and $u$ indicate the reference weight of the historical payoff of to himself and his neighbors is different.

As can be seen from fig. 6(a), with the decrease of the weight of self-reflection payoff, the level of group cooperation shows a trend of first rising and then decreasing under different levels of betrayal and temptation. When the proportion of 


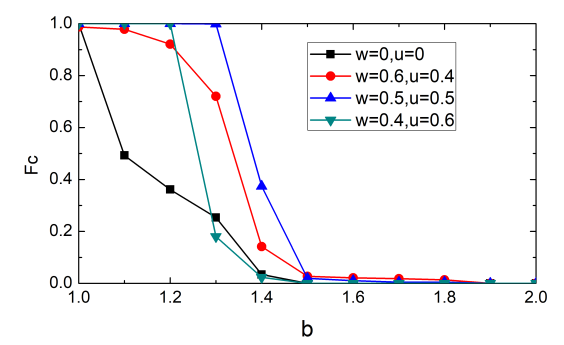

(a) small world network

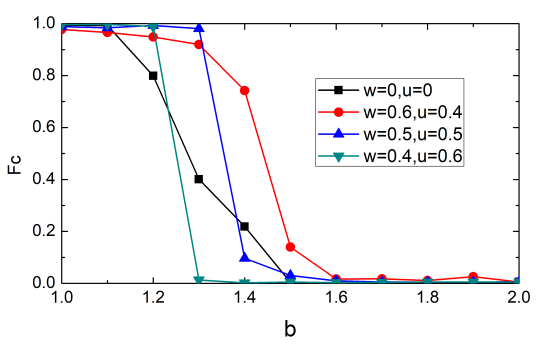

(b) random network and

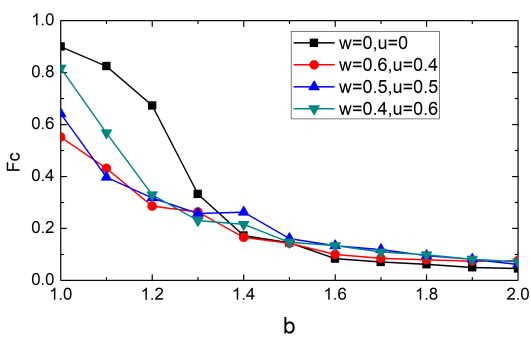

(c) scale-free network

Figure 6. The influence of payoff reflection mechanism on the level of cooperation in other networks.

self-reflection payoff and learning reflection payoff is the same, the cooperation level of the evolutionary group can be relatively optimal under different betrayal temptations, which is similar to the conclusion of this article on the grid network. The same proportion reflects the historical payoff of oneself and his neighbors, which can raise the level of group cooperation. However, it can be seen that the existence of the payoff reflection mechanism does not always enhance the cooperation level of the group. When the self-reflection parameter $w$ is small, the level of group cooperation can maintain a high proportion. However, when the betrayal temptation is greater than 1.3, the group cooperation level under the payoff reflection mechanism is lower than that of the traditional model, which is different from the experimental results on the grid network. When w is large, the level of group cooperation under the low temptation of betrayal is lower than that of the traditional model, and the level of cooperation under the high temptation of betrayal is higher than that of other situations. Through experiments on the small-world network model, it can be seen that the situation where the proportion of self-reflection and learning-reflection is not much different is still the best choice to improve the level of group cooperation. However, due to the influence of the network structure, it is no longer possible to maintain the number of collaborators under the temptation of high betrayal by adjusting the value of $w$ and $u$.

The change of cooperation level on the random network with the temptation of betrayal increases is shown in fig. 6(b). Changes in the level of cooperation are different from small world networks. With the decrease of self-reflection payoff parameter $\mathrm{w}$, the level of group cooperation has shown a decreasing trend. The larger self-reflection payoff parameter $\mathrm{w}$ will make the cooperation level slightly higher under the premise of less temptation of betrayal, but with the decrease of w, the cooperation level under the higher temptation of betrayal will be lower than the traditional model. So on the whole, the best-performing weight of self-reflection and learning-reflection reflection is $w=0.6, u=0.4$. It can be seen that in the random network, when the reference weight of the historical payoff of the oneself and his neighbors is appropriate, the payoff reflection mechanism can still have a positive impact on the improvement of the group cooperation level. However, when the weight of the two are similar, the group cooperation level cannot be optimally, and the greater reliance of the game individual on their own historical benefits will make the cooperation level greater.

From fig. 6(c), we can found that when the betrayal temptation is relatively small, the cooperation level of the traditional model is always the highest, that is, the payoff reflection mechanism suppresses the increase in the cooperation level of the group in the scale-free network. When the temptation of the betrayal is large, the payoff reflection mechanism slightly improves the level of group cooperation, but when the self-reflection payoff parameter $\mathrm{w}$ and learning-reflection payoff parameter $\mathrm{u}$ change, the level of cooperation does not change much. Overall, the payoff reflection mechanism cannot effectively promote the improvement of group cooperation level in scale-free network.

Through studies the impact of payoff reflection mechanism on group cooperation level in small world network, random network and scale-free network. It is found that the payoff reflection mechanism will have a different impact on group cooperation level under different network structure. In the small world network and random network, after introducing the payoff reflection mechanism, when the self-reflection payoff parameter $\mathrm{w}$ and learning reflection payoff parameter $\mathrm{u}$ have appropriate values, both can effectively promote the level of group cooperation. In the small world network, when the value of $\mathrm{w}$ and $\mathrm{u}$ are not much different, the level of group cooperation presents an optimal change. However, in the random network, when $w$ is large, the level of group cooperation presents an optimal change. Introducing the payoff reflection mechanism in scale free networks cannot effectively promote the improvement of the cooperation level of the group. When the temptation of betrayal is small, it will inhibit the size of the cooperative group.

\section{Discussion}

We have studied the synergistic effects of historical strategies and corresponding game payoff on current strategy learning, and proposed a payoff reflection mechanism. In the research on the impact of the payoff reflection mechanism on the evolution of cooperation, it is found that the payoff reflection mechanism can effectively promote the improvement of the level of group 
cooperation. This work also studies the impact of memory interval length $\mathrm{M}$ and payoff reflection parameter $w$ and $u$ on the evolution of cooperation. When the memory ability is different, the value of the payoff reflection parameter that can maximize the group cooperation level is different. The enhancement of memory ability will make the evolution of cooperation more gradual. The weight of the individual's self-reflection payoff in the game is inversely proportional to the level of group cooperation. In the last, we studied the impact of payoff reflection mechanism on group cooperation level on other networks, and find that introducing this mechanism in small world network and random network can effectively promote the improvement of cooperation level. However, in scale-free networks, even the size of cooperative groups will be inhibited. In general, our results show that the payoff reflection mechanism proposed in this paper has a very obvious effect on the improvement of group cooperation level.

\section{Methods}

This work mainly focuses on the study of strategy learning steps in evolutionary games. The game model uses the weak prisoner's dilemma model, and randomly selects neighbors for strategy learning. When the game individual chooses to learn the neighbor, the probability of learning the opponent's strategy is calculated by the deformed Fermi rule. Optimize the method of calculating the payoff of game individuals and neighbors in the traditional Fermi rule. Whether it is the payoff of game individuals or the payoff of selected neighbors, it will be affected by historical strategies and their payoff. If the payoff of current strategy is higher in the individual's historical behavior, the current strategy optimization payoff of the individual at the current time step is higher, which is higher than the benefit of the memoryless state. The following will introduce the game model and the deformed Fermi rule.

\begin{tabular}{lccc}
\hline \multirow{2}{*}{ Game individual and strategy } & \multicolumn{2}{c}{ Individual j } \\
& & $\mathrm{C}$ & $\mathrm{D}$ \\
\hline \multirow{2}{*}{ Individual i } & $\mathrm{C}$ & $\mathrm{R}, \mathrm{R}$ & $\mathrm{S}, \mathrm{T}$ \\
& $\mathrm{D}$ & $\mathrm{T}, \mathrm{S}$ & $\mathrm{P}, \mathrm{P}$ \\
\hline
\end{tabular}

Table 1. $2 \times 2$ symmetric game payoff.

In a $2 \times 2$ symmetric game, each game individual can choose a cooperative strategy (C) or a betrayal strategy (D). The profit matrix of both parties in each state game is shown in table 1 .

The classic prisoner's dilemma game must satisfy both conditions $T>R>P>S$ and $2 R>T+S$. In order to better control the variables, we study the influence of payoff reflection mechanism on the level of group cooperation. This work uses the Nowak and May's weak prisoner's dilemma game model, sets $T=b, R=1, P=S=0$ and $1<b<2$. Cooperation strategy is expressed as $S_{C}=(1,0)^{T}$, and the betrayal strategy is represented by $S_{C}=(0,1)^{T}$, and the payoff matrix is as follows.

$$
\begin{array}{ll}
\mathrm{C} & \mathrm{D} \\
\mathrm{D} & \left(\begin{array}{ll}
1 & 0 \\
b & 0
\end{array}\right)
\end{array}
$$

The payoff $\mathrm{U}$ of game individual $\mathrm{i}$ at each time step is calculated by eq. 1, where $\mathrm{n}$ represents the number of neighbors of the game individual.

$$
U=\sum_{j=1}^{N} S_{i}^{T} A S_{j}
$$

The neighbor selection strategy adopts the random selection in the traditional model, and the improved Fermi rule is used to calculate the probability when the learning object of the strategy is determined. The probability $\mathrm{W}$ of game individual i learning the strategy of game individual $\mathrm{j}$ is calculated by eq. 2 .

$$
W=\frac{1}{\left.1+\exp \left[P_{i}-P_{j}\right] / K\right]}
$$

Where $\mathrm{K}$ takes the value 0.1 , and $P_{i}$ and $P_{j}$ are the optimized payoff of the game individual $\mathrm{i}$ and his game neighbor $\mathrm{j}$ after reflecting on the historical payoff, and their final payoff will be affected by their own historical payoff. $P_{i}$ and $P_{j}$ are determined by eq. 3 and eq. 4 , respectively

$$
P_{i}=U_{i}+w * H_{i}
$$




$$
P_{j}=U_{j}+u * H_{j}
$$

The optimized payoff consists of two parts, namely the current strategic payoff and the reflective payoff. In eq. 3 and eq. 4 , $U_{i}$ and $U_{j}$ represent the payoff of the current strategy of game individual i and his game neighbor j, respectively. $H_{i}$ and $H_{j}$ represent the reflections of game individual $\mathrm{i}$ and his game neighbor $\mathrm{j}$, respectively. Parameters $\mathrm{w}$ and $\mathrm{u}$ are the reference coefficients of the reflection payoff of the game individual i and his game neighbor $j$, respectively, which are used to adjust the reference degree of the game individual to the historical payoff of himself and his neighbor. In each experiment, the values of $\mathrm{w}$ and $\mathrm{u}$ are fixed, $0 \leq w, u \leq 1$. When the two parameters are both set to 0 , the game individual does not reflect on payoff, and the evolutionary model returns to traditional modal. The reflection payoff $\mathrm{H}$ is calculated by eq. 5 .

$$
H=\left\{\begin{array}{l}
\sum_{n=1}^{M} P_{C}-\sum_{n=1}^{M} P_{D}, S=C \\
\sum_{n=1}^{M} P_{D}-\sum_{n=1}^{M} P_{C}, S=D
\end{array}\right.
$$

Where $S=C$ denotes that the strategy of the game individual at the current time step is cooperation, $S=D$ denotes that the strategy of the game individual at the current time step is betrayal. $P_{C}$ and $P_{D}$ respectively represent the historical payoff when the strategies in the individual memory of the game are cooperation and betrayal. Taking the strategy of current time step as the consideration strategy, the reflecting payoff is the difference of the total payoff of different strategies in historical memory. The value of the reflective payoff can reflect which strategy in the game individual's historical behavior will have a higher overall payoff on time. From the eq. 5, we can see that the higher the sum of the payoff of the same strategy in the memory and the current time step, the higher the reflection payoff, and the higher the optimization payoff. According to Fermi rule, the more likely the game individual will adhere to the current time step strategy.

\section{References}

1. Sanfey, A. G. Social decision-making: insights from game theory and neuroscience. Science 318, 598-602 (2007).

2. Gordon, D. M. The ecology of collective behavior in ants. Annu. review entomology (2019).

3. Bshary, R. \& Grutter, A. S. Image scoring and cooperation in a cleaner fish mutualism. Nature 441, 975-978 (2006).

4. Carattini, S., Levin, S. \& Tavoni, A. Cooperation in the climate commons. Rev. Environ. Econ. Policy 13, 227-247 (2019).

5. Perc, M. et al. Statistical physics of human cooperation. Phys. Reports 687, 1-51 (2017).

6. Pennisi, E. How did cooperative behavior evolve? Science 309, 93-93 (2005).

7. Macy, M. W. \& Flache, A. Learning dynamics in social dilemmas. Proc. Natl. Acad. Sci. 99, 7229-7236 (2002).

8. Hauser, O. P., Rand, D. G., Peysakhovich, A. \& Nowak, M. A. Cooperating with the future. Nature 511, 220-223 (2014).

9. Szabó, G. \& Fath, G. Evolutionary games on graphs. Phys. reports 446, 97-216 (2007).

10. Rapoport, A., Chammah, A. M. \& Orwant, C. J. Prisoner's dilemma: A study in conflict and cooperation, vol. 165 (University of Michigan press, 1965).

11. Sugden, R. et al. The economics of rights, co-operation and welfare (Springer, 2004).

12. Skyrms, B. The stag hunt and the evolution of social structure (Cambridge University Press, 2004).

13. Nowak, M. A. \& May, R. M. Evolutionary games and spatial chaos. Nature 359, 826-829 (1992).

14. Vukov, J., Szabó, G. \& Szolnoki, A. Evolutionary prisoner's dilemma game on newman-watts networks. Phys. Rev. E 77, 026109 (2008).

15. Qiu, T., Hadzibeganovic, T., Chen, G., Zhong, L.-X. \& Wu, X.-R. Cooperation in the snowdrift game on directed small-world networks under self-questioning and noisy conditions. Comput. Phys. Commun. 181, 2057-2062 (2010).

16. Zhang, Y., Aziz-Alaoui, M., Bertelle, C., Zhou, S. \& Wang, W. Emergence of cooperation in non-scale-free networks. J. Phys. A: Math. Theor. 47, 225003 (2014).

17. Rong, Z., Li, X. \& Wang, X. Roles of mixing patterns in cooperation on a scale-free networked game. Phys. Rev. E 76, 027101 (2007). 
18. Luo, C., Zhang, X., Liu, H. \& Shao, R. Cooperation in memory-based prisoner's dilemma game on interdependent networks. Phys. A: Stat. Mech. its Appl. 450, 560-569 (2016).

19. Qin, J., Chen, Y., Fu, W., Kang, Y. \& Perc, M. Neighborhood diversity promotes cooperation in social dilemmas. IEEE Access 6, 5003-5009 (2017).

20. Xie, Y., Chang, S., Yan, M., Zhang, Z. \& Wang, X. Environmental influences on cooperation in social dilemmas on networks. Phys. A: Stat. Mech. its Appl. 492, 2027-2033 (2018).

21. Shen, C., Chu, C., Shi, L., Perc, M. \& Wang, Z. Aspiration-based coevolution of link weight promotes cooperation in the spatial prisoner's dilemma game. Royal Soc. open science 5, 180199 (2018).

22. Huang, K., Zheng, X., Li, Z. \& Yang, Y. Understanding cooperative behavior based on the coevolution of game strategy and link weight. Sci. reports 5, 14783 (2015).

23. Cuesta, J. A., Gracia-Lázaro, C., Ferrer, A., Moreno, Y. \& Sánchez, A. Reputation drives cooperative behaviour and network formation in human groups. Sci. reports 5, 1-6 (2015).

24. Liu, J., Meng, H., Wang, W., Li, T. \& Yu, Y. Synergy punishment promotes cooperation in spatial public good game. Chaos, Solitons \& Fractals 109, 214-218 (2018).

25. Wu, Y., Zhang, B. \& Zhang, S. Probabilistic reward or punishment promotes cooperation in evolutionary games. Chaos, Solitons \& Fractals 103, 289-293 (2017).

26. Wang, W.-X., Ren, J., Chen, G. \& Wang, B.-H. Memory-based snowdrift game on networks. Phys. Rev. E 74, 056113 (2006).

27. Qin, S.-M., Chen, Y., Zhao, X.-Y. \& Shi, J. Effect of memory on the prisoner's dilemma game in a square lattice. Phys. Rev. E 78, 041129 (2008).

28. Wang, B. et al. Preferential selection based on payoff satisfaction and memory promotes cooperation in the spatial prisoner's dilemma games. EPL (Europhysics Lett. 129, 38002 (2020).

29. Juan, W., Li-Na, L., En-Zeng, D. \& Li, W. An improved fitness evaluation mechanism with memory in spatial prisoner's dilemma game on regular lattices. Commun. Theor. Phys. 59, 257 (2013).

30. Geng, Y. et al. Historical payoff promotes cooperation in voluntary prisoner's dilemma game. Chaos, Solitons \& Fractals 105, 145-149 (2017).

31. Deng, Z. et al. Historical payoff promotes cooperation in the prisoner's dilemma game. Chaos, Solitons \& Fractals 104, 1-5 (2017).

32. Jiang, L.-L., Perc, M., Wang, W.-X., Lai, Y.-C. \& Wang, B.-H. Impact of link deletions on public cooperation in scale-free networks. EPL (Europhysics Lett. 93, 40001 (2011).

\section{Acknowledgements}

This work is supported by the National Key Research and Development Program of China under grant No. 2018 YFB1003602.

\section{Author contributions}

B.W. and J.S. initiated the idea, B.H.,Q.D. and W.K. conducted the experiment(s). All authors analysed the results and reviewed the manuscript.

\section{Additional information}

Competing interests: The authors declare no competing interests. 
Figures

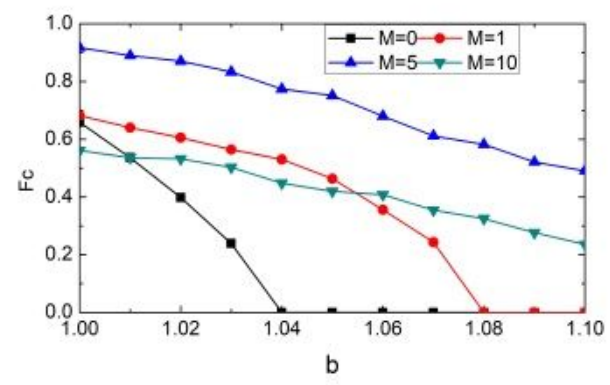

(a) selfish individuals

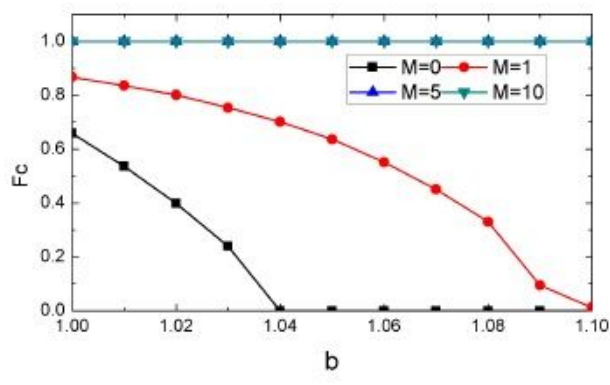

(b) conformity individuals

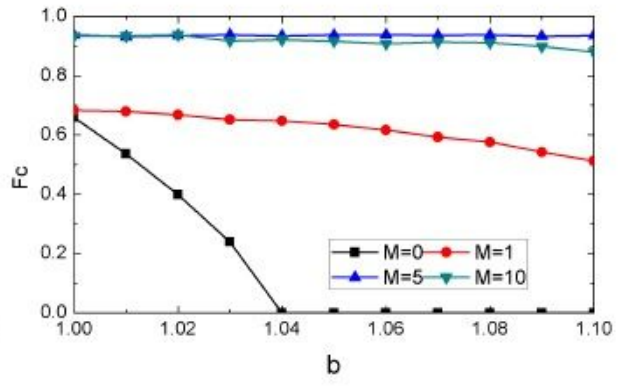

(c) ordinary individuals

Figure 1

Influence of payoff reflection mechanism on cooperation level.

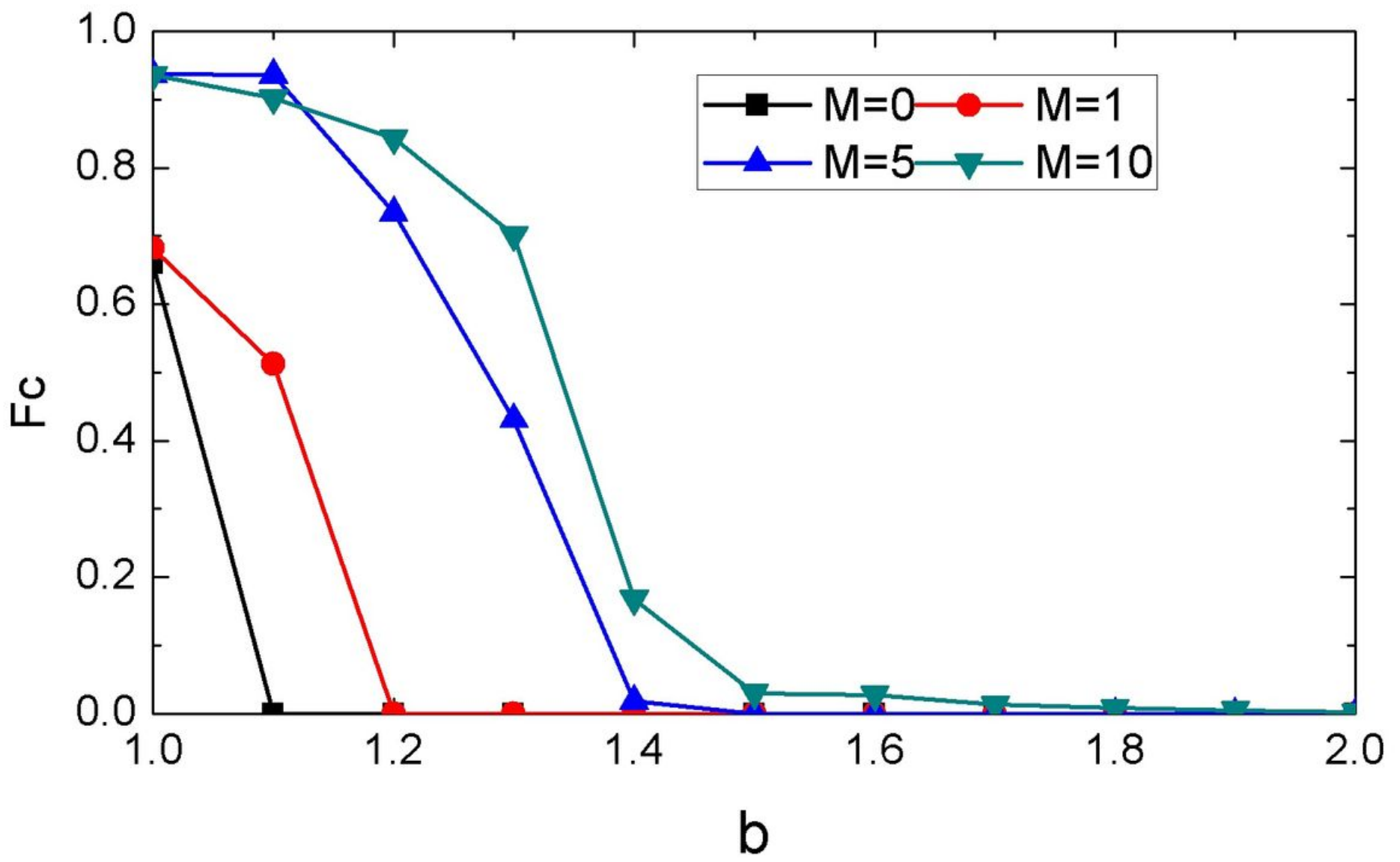

Figure 2

The influence of general individual's payoff reflection mechanism on the level of cooperation under high betrayal temptation. 


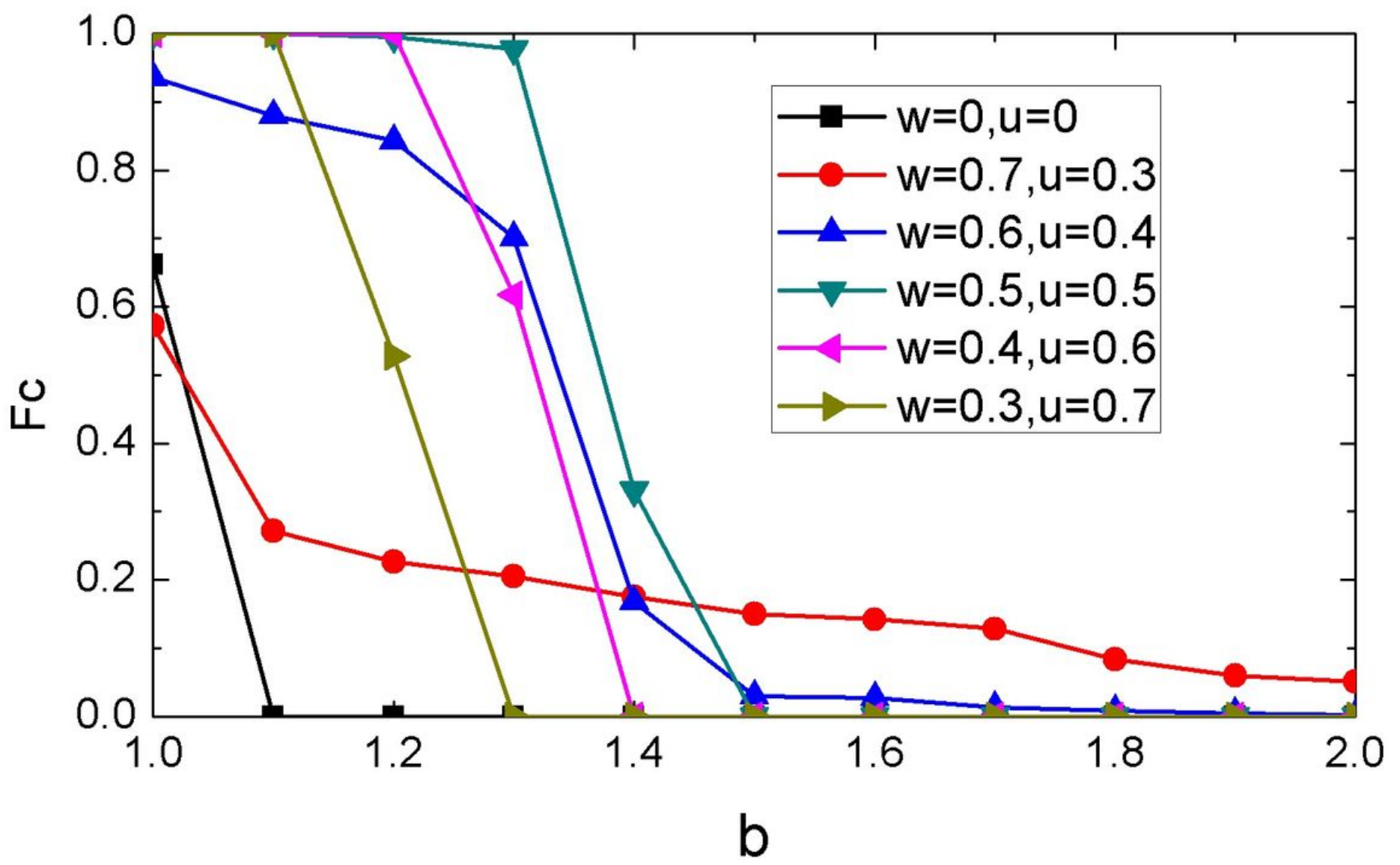

Figure 3

The impact of payoff reflection parameters on the level of cooperation 


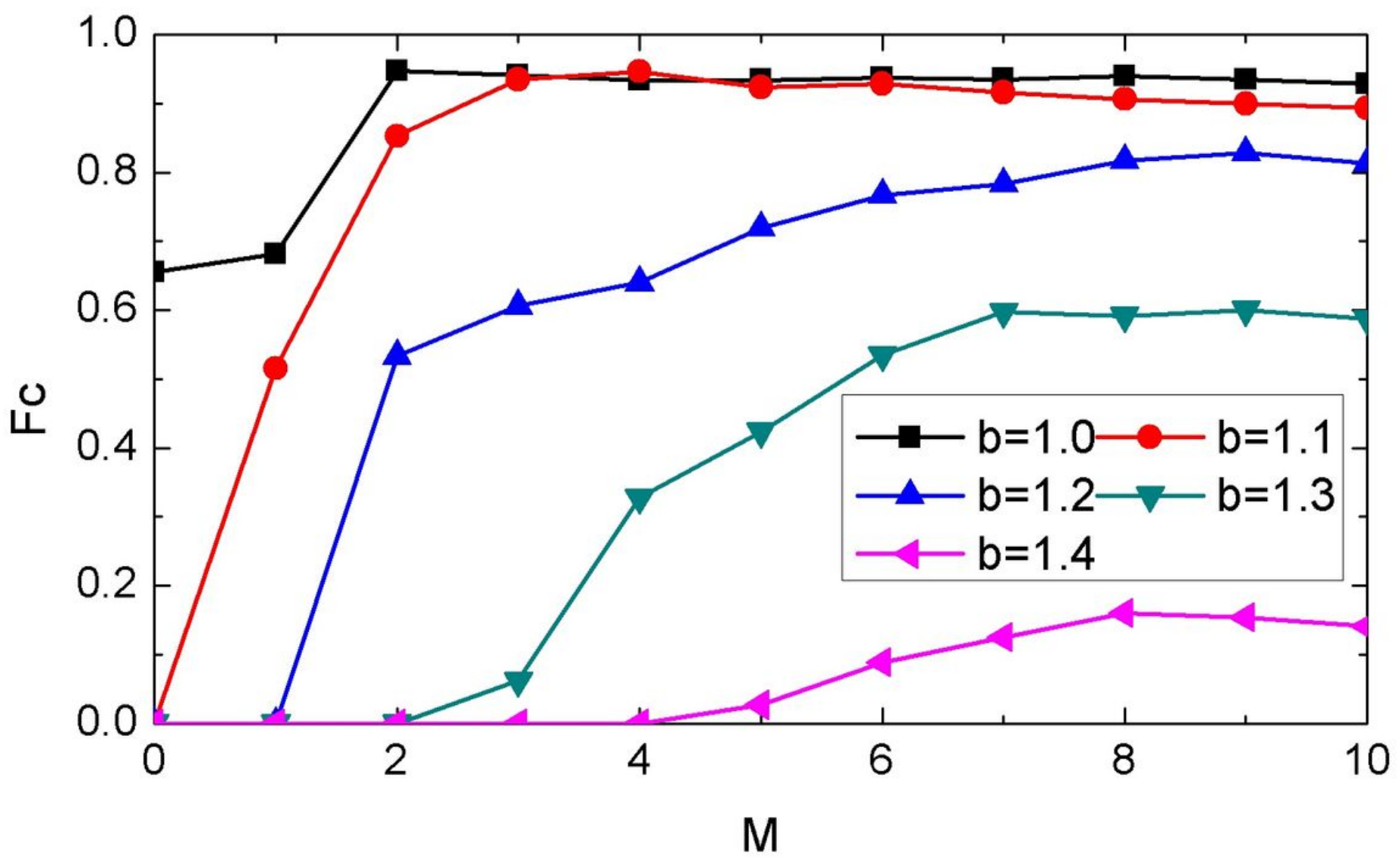

Figure 4

Influence of memory interval length on cooperation level.

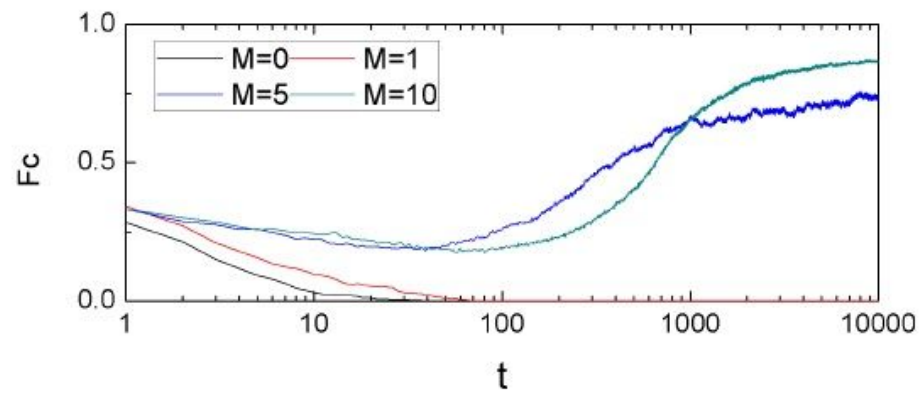

(a)

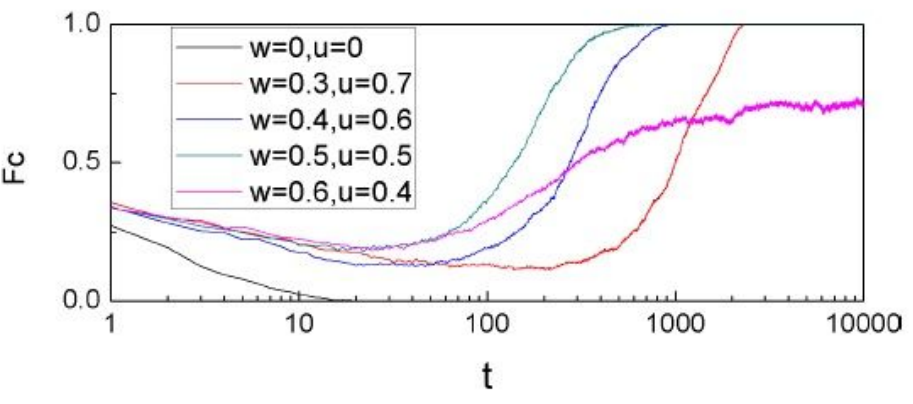

(b)

\section{Figure 5}

The influence of the parameters of the payoff reflection mechanism on the evolution of cooperation. 


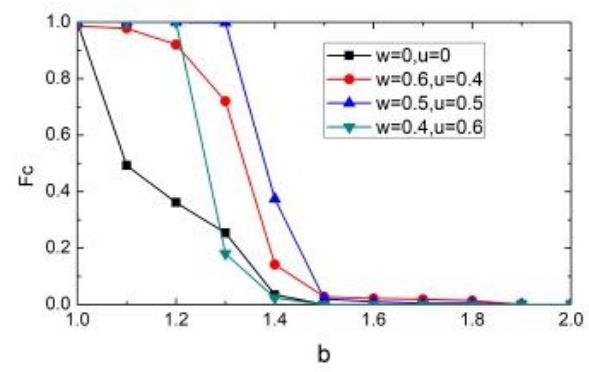

(a) small world network

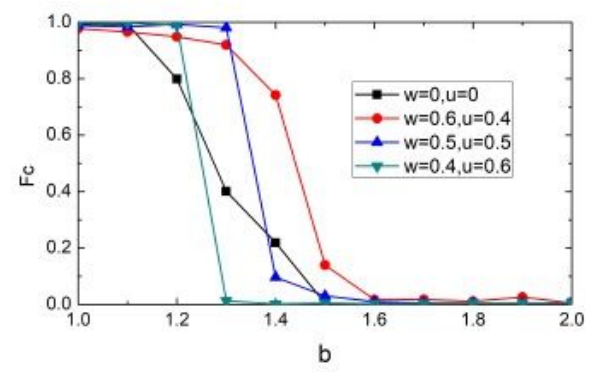

(b) random network and

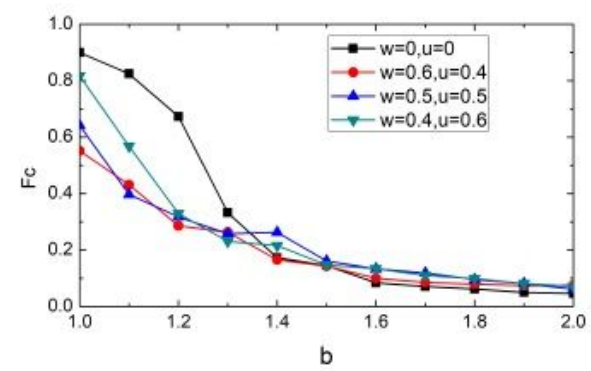

(c) scale-free network

\section{Figure 6}

The influence of payoff reflection mechanism on the level of cooperation in other networks. 